This is an Author's Accepted Manuscript of an article published in Memory (2014), 22:4, 390407, DOI: 10.1080/09658211.2013.795598 (copyright Taylor \& Francis), available online at: http://www.tandfonline.com/10.1080/09658211.2013.795598

\title{
The cultural life script of Qatar and across cultures: Effects of
}

$$
\text { gender and religion }
$$

\author{
Christina Lundsgaard Ottsen ${ }^{\mathrm{a}}$ and Dorthe Berntsen ${ }^{\mathrm{a}}$ \\ ${ }^{a}$ Center on Autobiographical Memory Research, Aarhus University, Denmark
}

Correspondence concerning this article should be addressed to Christina Lundsgaard Ottsen, CON AMORE, Department of Psychology and Behavioural Sciences, Aarhus University, Bartholins Allé 9, 8000 Aarhus C, Denmark, Tel.: +45 87165291, Email: $\underline{\text { col@psy.au.dk }}$

This is an Author's Accepted Manuscript of an article published in Memory (2014), 22:4, 390407, DOI: 10.1080/09658211.2013.795598 (copyright Taylor \& Francis), available online at: http://www.tandfonline.com/10.1080/09658211.2013.795598

Changes resulting from the publishing process, such as peer review, editing, corrections, structural formatting, and other quality control mechanisms may not be reflected in this document. Changes may have been made to this work since it was submitted for publication. 
This is an Author's Accepted Manuscript of an article published in Memory (2014), 22:4, 390407, DOI: 10.1080/09658211.2013.795598 (copyright Taylor \& Francis), available online at: http://www.tandfonline.com/10.1080/09658211.2013.795598

\begin{abstract}
Cultural life scripts (CLS) are culturally shared cognitive representations of the expected order and timing of important life events in a prototypical life. Through three studies, the data from Qatar were analysed and compared to previously collected data from Denmark, Turkey, and the US. In Study 1, we examined the CLS of Qatar in order to determine whether the clear segregation of men and women as well as the centrality of religion in this society would influence the CLS. Fifty-five Qatari undergraduates completed the standard CLS task, imaging a Qatari infant of their own as well as the opposite gender. In Study 2, important personal life story events were collected from eighty-three Qatari undergraduates in order to explore the overlap between remembered life events and CLS events. Study 3 was a reanalysis of the data from Denmark, Turkey, and the US. There was a considerable overlap of events across cultures, but we also found that the Qatari CLS showed more gender differences and contained more religious and positive events compared to the other three countries.
\end{abstract}

Keywords: autobiographical memory; cultural life script; gender; religion; reminiscence bump; childhood amnesia. 
This is an Author's Accepted Manuscript of an article published in Memory (2014), 22:4, 390407, DOI: 10.1080/09658211.2013.795598 (copyright Taylor \& Francis), available online at: http://www.tandfonline.com/10.1080/09658211.2013.795598

\section{The cultural life script of Qatar and across cultures: Effects of gender and religion}

To some extent, we are all a product of the culture in which we grow up. We are born into a world of common understandings (Geertz, 1966) and, from birth and onwards, we are expected to live through a certain number of life events. These events are expected to occur in a specific order and at prescribed periods during the life of the individual (Neugarten et al.,1965; Berntsen \& Rubin, 2004). The Cultural Life Script (CLS) reflects such shared expectations as to the order and timing of life events in a prototypical life (Berntsen \& Rubin, 2004). In the present studies, we examine the CLS and its relation to personal memories in Qatar and, therefore, a less WEIRD country than previously explored. The acronym WEIRD refers to the predominant use in behavioural sciences of subjects from Western, Educated, Industrialised, Rich, and Democratic countries. Such samples are problematic because various cross-cultural studies of perception, cooperation, categorisation, reasoning styles, self-concepts, and motivation show the WEIRD subjects to be an unusual minority and, therefore, among the least representative populations for generalising the human species (Henrich, Heine, \& Norenzayan, 2010). The CLS has mainly been studied across Western cultures (Bohn, 2010; Erdoğan et al., 2008; Habermas, 2007; Janssen \& Rubin, 2011; Rubin et al., 2009). All the studies have replicated the finding that most CLS events are expected to occur in young adulthood and that these events are rated dominantly positive (Berntsen \& Rubin, 2004). If these findings can be replicated in less WEIRD countries, it will not only strengthen the CLS theory as an explanation of the reminiscence bump, but will also provide further insight into the potential guiding function of CLS in the retrieval of autobiographical memories (Berntsen \& Rubin, 2003). To the extent differences are found, they will enhance our understanding of cultural variations in life expectations and values. In the following, we review the notion of the CLS, the possible 
This is an Author's Accepted Manuscript of an article published in Memory (2014), 22:4, 390407, DOI: 10.1080/09658211.2013.795598 (copyright Taylor \& Francis), available online at: http://www.tandfonline.com/10.1080/09658211.2013.795598

role of religion and gender, and the hypotheses that motivate the present series of studies.

\section{The notion of CLS}

Cultural narratives guide identity formation (McAdams, 1993, 1996) and cultural norms function as a measure of normal development (Bluck \& Habermas, 2000). The CLS display these norms in a given society. The notion of CLS combines script theory (Schank \& Abelson, 1977) with the idea of culturally sanctioned age norms (e.g. Neugarten, Moore, \& Lowe, 1965). Neugarten (1968) reported that major punctuation marks in adult life tend to be more social than biological. In support of this the CLS tends to include rather few events that follow a purely biological timing such as menarche, menopause, and death, instead mainly culturally sanctioned events such as marriage are featured. Likewise, the estimated prevalence of events is a relatively poor predictor for how frequently the events are recorded as typical life script events. The CLS is not formed by personal experience. It is passed down from generation to generation and stored in memory as semantic knowledge (Berntsen \& Rubin, 2004). A standard way to obtain a schema of such shared knowledge is to have people generate imaginary instances of the schema of interest (Rubin \& Kontis, 1983; Rubin, Stolzfus, \& Wall, 1991). With the use of open-ended instructions to generate the seven most important events in a prototypical life course, this is how the CLS is obtained (Berntsen \& Rubin, 2004).

Every culture has a prescribed timetable for the transitional events that people are expected to live through at specific times during their life course (Neugarten et al., 1965). The cultural representations of a typical life course can be considered as a timeline of important events that guides the individual both in life and memory (Rubin \& Berntsen, 2003). Most transitional events are expected to be positive and they often take place in young adulthood. The internalised script of these events acts as a template for recalling life 
This is an Author's Accepted Manuscript of an article published in Memory (2014), 22:4, 390407, DOI: 10.1080/09658211.2013.795598 (copyright Taylor \& Francis), available online at: http://www.tandfonline.com/10.1080/09658211.2013.795598

events from different life phases, according to the life script theory (Berntsen \& Rubin, 2004). Therefore, the CLS provides a plausible explanation for the overrepresentation of personal events between the ages between 15 and 30 that Rubin, Wetzler, and Nebes (1986) named "the bump". The CLS theory also offers an explanation as to why a request for emotionally positive, but not negative, memories shows a bump (Berntsen \& Rubin, 2002; Rubin \& Berntsen, 2003).

Although life scripts help to structure individual life stories, the two concepts are theoretically and empirically distinct (Berntsen \& Rubin, 2004). A CLS is a part of our generally shared semantic knowledge within a given culture. A personal life story is unique, individual, and part of our autobiographical knowledge. The individual life story includes both life script events (e.g., my wedding day) as well as deviations from the life script (e.g., why I never graduated college). A life story is measured by asking an individual to tell about his or her own life, whereas the CLS is measured by a request for events in a prototypical life within a given culture (Berntsen \& Rubin, 2002; Rubin \& Berntsen, 2003). The CLS has been studied in Denmark, Turkey, Germany, Malaysia, the Netherlands, and the US (Berntsen \& Rubin, 2004; Erdoğan et al., 2008; Habermas, 2007; Haque \& Hasking, 2010; Janssen \& Rubin, 2011; Rubin et al., 2009). Across cultures, the contents of the highly frequent event categories are quite similar and in general only minor cross-cultural differences have been found in the CLS. Notably, across cultures, a dominance of emotionally positive events from the period of young adulthood is seen.

\section{Religion viewed as a cultural system}

Culture can be defined as a historically transmitted pattern of meanings through which people develop their knowledge about and attitudes towards life. This pattern is embodied in symbols and the utilisations of these symbolic forms are cultural acts at social events. To 
This is an Author's Accepted Manuscript of an article published in Memory (2014), 22:4, 390407, DOI: 10.1080/09658211.2013.795598 (copyright Taylor \& Francis), available online at: http://www.tandfonline.com/10.1080/09658211.2013.795598

which extent these events will be religious depends on the effects of religion in a given society (Geertz, 1966). Religion can be regarded as a subset of culture (Hulsether, 2005) and, like culture, religion both reflects social order in a society and continuously shapes it (Geertz, 1966). Therefore, if cultural norms structure expectations to the contents and order of events in a typical life course, then religious traditions and rituals should be evident in the CLS of religious societies (Berntsen \& Rubin, 2004; Habermas \& Bluck, 2000). In support of this, a study of the CLS in a Muslim sample from Malaysia showed many events associated with religious activities (Haque \& Hasking, 2010). The study of the Malaysian CLS used the same emotion cues as used by Rubin and Berntsen (2003) in one of the preliminary studies that led to the CLS account of the reminiscence bump. Religion was less prevalent in the Turkish CLS (Erdoğan et al., 2008). This study used the same method as Study 2 in Berntsen and Rubin's (2004) study of the Danish CLS.

Erdoğan et al. (2008) found considerable cross-cultural similarity between the CLS of Denmark and the CLS of Turkey. Only a few events differed in content and valence showing Turkey to be a more collectivistic culture (Rubin et al., 2009). The marked resemblances across cultures could to some extent be due to the fact that the Turkish participants were students from the Boğaziçi University in Istanbul and, therefore, the Turkish sample is likely to be more WEIRD than people from the rural areas of Turkey. Subcultures within a country can have significantly different cultural values (Taras, Kirkman \& Steel, 2010). Therefore, the CLS might vary not only by country, but also by subcultures within a given country (Haque \& Hasking, 2010). Separate geographical regions, ethnicity, religion, or gender segregation could be the base of such subcultures.

\section{Gender difference and the CLS}


This is an Author's Accepted Manuscript of an article published in Memory (2014), 22:4, 390407, DOI: 10.1080/09658211.2013.795598 (copyright Taylor \& Francis), available online at: http://www.tandfonline.com/10.1080/09658211.2013.795598

No significant differences have been found across gender in terms of events, age or emotional valence. However, the study of the Turkish CLS is the only study directly designed to examine the effects of gender. In other studies, participants have generated a CLS for an infant of their own gender. In contrast, the Turkish study had a $2 \times 2$ design where all the participants filled out the standard CLS questionnaire imagining both the prototypical life of a female and a male infant in their own culture (Erdoğan et al., 2008). The participants generated almost the same event categories regardless of their own gender and they also estimated the age and emotion for these events in similar ways.

In gender segregated societies, men and women rarely interact (Bahry \& Marr, 2005). They live in a female and a male subculture, which might cause their cultural expectations of a normative life to differ. Should that be the case, then men and women in gender segregated societies might generate CLS that differ even though they are part of the same culture. The CLS could also differ across gender in another way. If people living in gender segregated societies have different cultural expectations for the male role contra the female role then they might generate a CLS for each gender role.

\section{The non-secular and gender segregated society of Qatar}

The state of Qatar is a small peninsula in the Arabian Gulf and is best known for its energy reserves and rapid economic expansion. Ruled by HH Sheikh Hamad bin Khalifa AlThani, this country is one of the least conservative emirates in the region (Clinton, 2011). However, Qatar is still a traditional Muslim society with deep cultural roots and daily life can be described as divided into two parallel societies - a male and a female one - with minimal interaction between men and women (Bahry \& Marr, 2005). At the state university the campus is gender divided. Public institutions, libraries, and amusement parks have special weekdays for women and children to visit wherein men are not allowed. Wedding 
This is an Author's Accepted Manuscript of an article published in Memory (2014), 22:4, 390407, DOI: 10.1080/09658211.2013.795598 (copyright Taylor \& Francis), available online at: http://www.tandfonline.com/10.1080/09658211.2013.795598

parties are kept in separate rooms - one for the bride and one for the groom. Women are not obligated, as men, to attend Friday Noon Prayer in the mosques, but when they do attend they are separated from the men. In general, girls are socialized to be more private in public than boys, and wearing a veil is viewed as a prerogative that protects women's right to see the world without being watched. At the same time, it signals both status and modesty (El Guindi, 2003). Since 1950, schooling has been free of charge in Qatar and the government encourages the education of both genders. By 1978, more girls than boys have been graduating from high school and today there is an imbalance in university enrolments with women outnumbering the men (Bahry \& Marr, 2005). The national development strategy of Qatar recognises the challenges posed in balancing continuous modernisation with the preservation of culture and traditions. Qatar eagerly invests in education and public health with the aim of playing a pivotal role at both the regional and international levels (Qatar general secretariat for development planning, 2011).

First and foremost, Qatar deviates from the countries previously studied because it is a gender divided society. The clear segregation of men and women in this Muslim country presents an ideal opportunity for exploring gender differences and the effects of religion. Qatar is not Western and as the Emir still appoints the government without a free election, it cannot be called a democracy (Rathmell, 2000). Qatar is extremely wealthy, it focuses on higher education, and it has recently been through a rapid process of industrialisation and, therefore, to some extent it fulfils three out of five of the conditions of being WEIRD.

However, these three features are newly acquired and have only defined Qatar within the last one or two generations (Looney, 1992).

\section{The present studies}


This is an Author's Accepted Manuscript of an article published in Memory (2014), 22:4, 390407, DOI: 10.1080/09658211.2013.795598 (copyright Taylor \& Francis), available online at:

\section{http://www.tandfonline.com/10.1080/09658211.2013.795598}

As reviewed in the introduction, the previous studies of CLS show many similarities and only a few differences across both culture and gender. The present studies examine if these similarities are still evident when exploring the CLS of Qatar, a non-secular society with a clear segregation of men and women.

In Study 1, we examine the CLS of Qatar. We hypothesise that the Qatari CLS, following the previous studies, will be dominated by positive events happing in young adulthood, but we also expect the CLS of Qatar to show signs of cultural traits such as religion and gender segregation.

In Study 2, we follow up on Study 1 by exploring the important life story events of the Qataris in order to examine the extent to which the event categories overlap with the CLS. We hypothesise that the specific cultural characteristics of the CLS will be evident in the life stories and that an overlap between the life story events and CLS events will be considerable but far from complete.

In Study 3, we compare the data from Qatar with a reanalysis of the data from Denmark, Turkey, and the US in order to examine if there are cross-cultural differences in the CLS. We hypothesise that a reanalysis using the same variables as used in Study 1 will confirm the Qatari CLS to be characterised by religion more than the life scripts from more secular and less gender segregated societies. For the reanalysis, we chose studies from countries which all differ from Qatar in theoretically relevant ways. Turkey was chosen because it was relevant to compare Qatar, a society where the political system is intertwined with religion (Hamzeh, 1994), to a more secular Muslim society, where no gender differences have been found in the CLS (Erdoğan et al., 2008). In addition, we chose two extremely WEIRD countries from two different continents: Denmark and the US. Denmark is traditionally Protestant, but functionally it is one of the least religious societies in the 
This is an Author's Accepted Manuscript of an article published in Memory (2014), 22:4, 390407, DOI: 10.1080/09658211.2013.795598 (copyright Taylor \& Francis), available online at: http://www.tandfonline.com/10.1080/09658211.2013.795598

world, whereas the US is one of the most multi-religious societies in the world (Zuckerman, 2008). The data from Qatar were collected by the first author during the years she lived there. The data on the CLS from Denmark, Turkey, and the US were reanalysed from the previous studies (Berntsen \& Rubin, 2004; Erdoğan et al., 2008; Rubin et al., 2009).

\section{STUDY 1}

The aim of Study 1 was to examine the CLS of Qatar. We hypothesised that the clear segregation of men and women as well as the centrality of religion in Qatar would affect the CLS. Consistent with previous studies (Berntsen \& Rubin, 2004; Bohn, 2010; Janssen \& Rubin, 2011; Rubin et al., 2009), the first analyses are based on the participants' answers for their own gender, whereas the section comparing gender follows the Turkish design using each participant's answer for both genders (Erdoğan et al., 2008).

\section{Method}

\section{Participants}

Sixty-three undergraduates recruited from four classes at Qatar University participated. Eight were eliminated because they left most of the questions unanswered or had a nationality from outside the Gulf region. The final number of 55 participants included 31 women $\left(\mathrm{M}_{\mathrm{age}}=21.4, \mathrm{SD}=1.7\right)$ and 24 men $\left(\mathrm{M}_{\mathrm{age}}=24.7, \mathrm{SD}=4.3\right)$ with an age range of $19-44$ years.

\section{Material}

The CLS task, as developed by Berntsen and Rubin (2004), was translated into Arabic. The instructions for the study were printed on the first page of the questionnaire. In an English translation, they read as follows: "Imagine a quite ordinary infant. It cannot be a specific infant that you know, but a prototypical infant in our culture with a quite ordinary 
This is an Author's Accepted Manuscript of an article published in Memory (2014), 22:4, 390407, DOI: 10.1080/09658211.2013.795598 (copyright Taylor \& Francis), available online at:

\section{http://www.tandfonline.com/10.1080/09658211.2013.795598}

life course ahead. Your task is to write down the seven most important events that you imagine are highly likely to take place in this prototypical infant's life. Write the events in the same order as they come to your mind. Give each event a short title that specifies its content." On the second page, the participants wrote down their answers for both genders.

\section{Design}

The participants answered the standard CLS task (Berntsen \& Rubin, 2004) for both a male and a female infant. This $2 \times 2$ design (women's versus men's answers $\mathrm{x}$ female versus male CLS) corresponded to Erdoğan et al.'s (2008) study of the Turkish CLS. The design enables two between-subject analyses: one compared men and women's different expectations to the Qatari female role and another compared their expectations to the Qatari male role. In addition, it makes two within-subjects analyses possible: one compared the women's expectations to both cultural gender roles and another compared the men's expectations to both gender roles.

\section{Procedure}

For each recorded event, the participants estimated the age at which the event was expected to take place and they rated whether they perceived the event to be emotionally positive or negative on a scale from -3 to +3 . The participants filled out the questionnaire during class. Each data collection session started with a short presentation of the concept of the CLS. Then, a local teacher read the instructions aloud in Arabic and the estimation of emotional valence was explained. The participants were reminded that they were to imagine the entire life course of both the female and the male infants and it was emphasised that there were no correct or incorrect answers. The participants were welcome to ask questions in both English and Arabic during the task. After collecting the data, all the questionnaires were translated into English with help from a local translator. 
This is an Author's Accepted Manuscript of an article published in Memory (2014), 22:4, 390407, DOI: 10.1080/09658211.2013.795598 (copyright Taylor \& Francis), available online at: http://www.tandfonline.com/10.1080/09658211.2013.795598

\section{Dependent variables}

An overview of the dependent variables is shown in Table 1. The data were analysed with regard to emotional valence, age estimate and whether the events were categorised as "other" and not a CLS event (referred to as idiosyncratic events). These variables derive from previous work (Bohn, 2010; Rubin et al., 2009). Furthermore, three new variables were generated with the purpose of the present study, all concerning the content of the recorded events. These addressed whether the events were religious ("Religious events"), whether they were found across the four countries ("Cross-cultural events"), or whether they were specific to Qatar (“Country specific events”), see Table 1.

\section{Coding}

Unanimous event categories from the three previous studies in Denmark, Turkey, and the US were used as a guideline for the categorisation of the events (Berntsen \& Rubin, 2004; Erdoğan et al., 2008; Rubin et al., 2009). A local translator was consulted for cultural explanations before 16 new event categories were created. The new categories primarily regarded religion and culture, e.g. "Hajj - the pilgrimage to Mecca" and "Women's independence" $\left(\mathrm{M}_{\mathrm{age}}=45\right.$ years - the latter referring to a time after the childbearing years where women have a central role in the family (Shweder, 1998). Consistent with the Danish CLS, the CLS of Qatar was generated by using the answers that each participant gave for their own gender, respectively. All the events mentioned by at least $4 \%$ of the participants were accepted following prior studies' lower limit of 4 coinciding events per 100 participants (Berntsen \& Rubin, 2004; Erdoğan et al., 2008; Rubin et al., 2009; Bohn, 2010). The events were classified according to 36 categories (Table 2). After the scoring of the data, half of the data were scored again independently by a research assistant who was blind 
This is an Author's Accepted Manuscript of an article published in Memory (2014), 22:4, 390407, DOI: 10.1080/09658211.2013.795598 (copyright Taylor \& Francis), available online at: http://www.tandfonline.com/10.1080/09658211.2013.795598

to the original coding. The agreement was on $79 \%$ of all the events (Cronbach's alpha $=$ $.85)$.

\section{Results}

\section{Characteristics of the CLS of Qatar}

Table 2 shows the CLS of Qatar including sixteen country specific event categories marked with a "Q". The country specific events are unique to Qatar in the sense that they are not mentioned in the CLS of Denmark, Turkey, and the US. The Qatari CLS have nine religious event categories, which equals $25 \%$ of all the categories in the CLS (Table $2-$ marked R). The Qataris show high agreement on the religious age estimates measured in years (SDs < 1.7), except for "Hajj/pilgrimage" (SD = 4.2), which can be done at all times during adult life. Most of the religious categories are estimated to happen in the first decade of life and all are rated as highly positive, except menarche ${ }^{1}\left(\mathrm{M}_{\mathrm{valence}}>1.00\right)$. In general, the CLS of Qatar is characterised by the expectations of positive life events and consistent with previous studies (Berntsen \& Rubin, 2004; Erdoğan et al., 2008; Rubin et al., 2009; Bohn, 2010) lower standard deviations show stronger agreement about the timing of positive events (SDs < 12.7) compared to negative events (SDs < 26.1). With three negative categories, only $8 \%$ of the categories in the Qatari CLS have a negative mean valence. Event categories not mentioned by Qataris, but present in most other CLS, are "Fall in love", “Parents' death", and "Divorce" (Berntsen \& Rubin, 2004; Erdoğan et al., 2008; Janssen \& Rubin, 2011). The top ten events in the Qatari CLS mainly consist of cross-cultural event categories, that is events that are recorded in the CLS of Qatar as well as in the CLS of Denmark, the US, and Turkey (Table 2 - events marked Cc). In total, thirteen event categories are shared across these cultures. The main features of the Qatari CLS support findings from the previous studies of CLS, showing many similarities across cultures (Bohn 
This is an Author's Accepted Manuscript of an article published in Memory (2014), 22:4, 390407, DOI: 10.1080/09658211.2013.795598 (copyright Taylor \& Francis), available online at: http://www.tandfonline.com/10.1080/09658211.2013.795598

\& Berntsen, 2011; Erdoğan et al., 2008; Haque \& Hasking, 2010; Janssen \& Rubin, 2011; Rubin et al., 2009).

\section{Comparison by gender in Qatar}

The following analyses are based on all the data from Qatar and not just on the answers that the participants gave regarding the CLS of their own gender. As in Erdoğan et al.'s study (2008), the data yields four CLS (Table 3) showing both the Qatari men and the Qatari women's expectations of the male role versus the female role in Qatar. When comparing the top 10 events in the four Qatari CLS, the findings resemble Erdoğan et al.'s findings by showing more similarities than differences between the perception of the male role and the female role. However, notable differences were found between-subjects, especially with regard to religious events. The Qatari men generated five different religious event categories while the women generated only one.

To examine the characteristics of how the CLS for male and female targets may interact with the gender of the participants as well as to explore potential main effects, a series of two-way ANOVAs were conducted. Each analysis had gender as between-subject variable (men's answers versus women's answers) and one of the variables, e.g., “idiosyncrasy”, as a repeated measure factor with two levels (male CLS versus female CLS). As shown in Table 4, a main effect of gendered CLS (how the male and female role is viewed) was found only for "religious events" with the male CLS including more religious events, whereas the main effects of gender (men's answers vs. women's answers) were found for "cross-cultural events", "country specific events", "religious events", and "age estimates" (Table 4). Compared to Qatari women, the men's answers included fewer crosscultural events, but more country specific and more religious events. The only interaction found regarded religious events. Follow up t-tests showed that Qatari men generated 
This is an Author's Accepted Manuscript of an article published in Memory (2014), 22:4, 390407, DOI: 10.1080/09658211.2013.795598 (copyright Taylor \& Francis), available online at: http://www.tandfonline.com/10.1080/09658211.2013.795598

significantly more religious events in both the male, $t(31.8)=4.787, p=.001, d=.65$ and the female CLS, $t(33.1)=2.733, p=.01, d=.43$ compared to the Qatari women, but this effect was largest for the male CLS. The main effect of age estimates showed the men to generate lower age estimates in general. This latter effect might be due to the low age estimates of the religious event categories since religious events were predominantly mentioned by Qatari men.

In general, the comparison of CLS across gender showed almost no difference within-subjects as to how the male and female role is viewed. The between-subjects analyses, however, showed the Qatari men to focus less on cross-cultural events and more on events specific to Qatar, including religious events, than the Qatari women.

\section{The positive CLS bump}

The distribution of the Qataris' age estimates for positive events showed a bump between the ages 15-29, while the neutral and negative events showed a more even distribution throughout the life span (Figure 1). This shows the Qataris' estimation of emotional valence and the life span distribution of events to be consistent with previous studies (Berntsen \& Rubin, 2004; Erdoğan et al., 2008; Janssen \& Rubin, 2011; Rubin et al., 2009). Of all the events generated in the Qatari CLS - a script of the full lifespan - 50\% were estimated to happen in the short period between the ages 15-29 and these events formed a peak that mirrored the standard reminiscence bump for autobiographical memory (Berntsen \& Rubin, 2002).

In contrast to previous studies, the distribution of positive events in the Qatari CLS showed a markedly elevated starting point. The early age estimates of the religious events suggest that this first decade peak is partly due to religion. Islamic events and cultural customs related to Islam account for nine out of fourteen event categories in the first teen 
This is an Author's Accepted Manuscript of an article published in Memory (2014), 22:4, 390407, DOI: 10.1080/09658211.2013.795598 (copyright Taylor \& Francis), available online at: http://www.tandfonline.com/10.1080/09658211.2013.795598

years of life (Learning the Quran, Islamic birth rituals, Circumcision, Learn Islamic values and moral, Learn to pray, Go to the mosque, Learn fasting, and Gender divided social skills ${ }^{2}$ ). Even though these nine categories are not highly frequent, they still account for $32 \%$ of all positive events that the Qataris mentioned for this period of life. The rest of this elevated starting point mainly consists of biological and educational event categories such as "Begin Walking", “Own Birth" and "Begin School”. Since the religious events are rated as highly positive (Table 2 - marked R), this indicates that the elevated frequency of events in the first decade of the Qatari CLS is due to religious events and that the first decade of life is a time for learning Muslim tradition, behaviour, and values.

\section{Discussion}

Study 1 showed that the CLS of Qatar was characterised by positive and religious events happening early in life. Some of the low frequent religious events were only mentioned by a couple of people and, therefore, these events could be idiosyncratic. Because there was a limit of recording only seven important life events, the participants are not likely to agree on all events they recorded as the most important. This does, however, not mean that they will not recognise the CLS events mentioned by other participants as shared cultural knowledge (Berntsen \& Rubin, 2004). Following previous studies, the Qataris showed high agreement about the timing of positive events. This agreement of temporal norms across the participants also indicates a culturally shared conception of these events (Rubin \& Berntsen, 2003). Most of the positive events were expected to occur between the ages $15-30$. Interestingly, the bump period was not the only period dominated by positive events in the Qatari CLS. The Qataris frequently generated events with low age estimates and these events were also predominantly positive. The focus on positive religious events that are expected to happen in the first decade of life was especially high in the CLS 
This is an Author's Accepted Manuscript of an article published in Memory (2014), 22:4, 390407, DOI: 10.1080/09658211.2013.795598 (copyright Taylor \& Francis), available online at: http://www.tandfonline.com/10.1080/09658211.2013.795598

generated by men, suggesting that these participants emphasised religion as a model for life with a detailed script for upbringing in a Muslim culture (Geertz, 1966).

Gender difference was most evident in the between-subjects analyses of the men's expectations versus the women's expectations towards a normative life in the Qatari culture. The Qatari men focused more on events specific to their own country, while women focused on events shared across cultures. Only minor gender differences were found within-subjects with regard to the CLS generated for the male role versus the CLS generated for the female role and the only significant difference concerned religion. Both men and women mentioned religious events to a higher extent for the male role. This effect was especially pronounced among the male participants.

To sum up, consistent with our hypotheses, the CLS of Qatar was dominated by positive events happing in young adulthood. This is in accordance with previous studies of the CLS but, in contrast to these studies, the CLS of Qatar was also characterised by religious events and gender differences. The gender differences were mainly found betweensubjects, possibly portraying a male and a female subculture in this segregated society. Study 2 was conducted to explore if these same characteristics were evident in the personal life story events of the Qataris.

\section{STUDY 2}

While the CLS is a culturally shared part of our semantic knowledge, a life story is an individual and unique part of our autobiographical memory. In this way, the two concepts are theoretically and empirically distinct (Berntsen \& Rubin, 2002; Rubin \& Berntsen, 2003). When presenting the CLS theory, Berntsen and Rubin (2004) predicted a partial overlap between events in the CLS and important life story events. A follow-up study found 
This is an Author's Accepted Manuscript of an article published in Memory (2014), 22:4, 390407, DOI: 10.1080/09658211.2013.795598 (copyright Taylor \& Francis), available online at: http://www.tandfonline.com/10.1080/09658211.2013.795598

the degree of overlap to vary across cultures, i.e., between Denmark and the US (Rubin et al., 2009). In Study 2, we examined life story events of Qatari students to compare expected CLS events with important life story events from a non-Western population.

\section{Method}

\section{Participants}

Participants were 54 undergraduates from Qatar University (two female classes and one male class) and 30 undergraduates from a mixed class at Texas A\&M University’s branch in Qatar. One participant was eliminated on account of being a foreigner in Qatar on a scholarship. The final number of 83 participants included 47 women $\left(\mathrm{M}_{\mathrm{age}}=20.7\right.$ years, $\mathrm{SD}=2.1)$ and 36 men $\left(\mathrm{M}_{\mathrm{age}}=21.7, \mathrm{SD}=3.5\right)$ with an age range of 17-30 years. Among the participants, $21 \%$ were married and $11 \%$ had at least one child.

\section{Design}

All the participants generated seven important events that they would include in their own personal life. Thus, the design of the life story task resembled the standard CLS task (Berntsen \& Rubin, 2004), but instead of answering for an imaginary infant the participants wrote down the seven most important events from their own life, from their birth to their current age.

\section{Material}

The questionnaire regarding the life story task followed previous studies (Bohn, 2010; Rubin et al., 2009). It was translated into Arabic by a local translator and the instructions for the study were printed on the questionnaire. In an English translation, they read as follows: "This study deals with your personal life story. Your task is to decide which events are the most central to the story of your own life. It has to be events that you have personally experienced. Imagine that you are to tell your personal life story to a new friend, whom you 
This is an Author's Accepted Manuscript of an article published in Memory (2014), 22:4, 390407, DOI: 10.1080/09658211.2013.795598 (copyright Taylor \& Francis), available online at: http://www.tandfonline.com/10.1080/09658211.2013.795598

have just met and who does not know anything about your past. It is a friend with whom you are absolutely confident and with whom you can be completely honest. Your task is to note seven memories about events from your own personal life (from your birth to your present age) that you think are most central to your life story. Write the events in the same order as they come to your mind".

\section{Procedure}

For each of the seven events, the participants wrote down at what age the event took place and the emotional valence of the event following the procedure from Study 1. After data collection, all the responses were translated into English by a professional translator.

\section{Dependent variables}

The dependent variables in Study 2 followed those in Study 1 with minor changes (see Table 1). The variable "age estimates" was disregarded since it only concerned CLS events. The variable "life story typicality" was introduced. It measures how closely the participants follow the CLS norms of their own culture when generating life story events. Instead of script valence, the dependent variable measuring emotional valence in Study 2 was "story valence", referring to the remembered emotion in relation to the experienced events reported in life stories.

\section{Coding}

Previous studies of life story memories using the same method were used as guidelines for the categorisation of the Qatari life story events. New categories were formed when the same event was mentioned by at least $4 \%$ of the participants (Bohn, 2010; Rubin et al., 2009). The procedure was performed twice - once by a research assistant and once by the first author. The categories agreed upon were chosen. This resulted in a total of 34 event categories of which 19 were newly generated. After the formation of the categories, two 
This is an Author's Accepted Manuscript of an article published in Memory (2014), 22:4, 390407, DOI: 10.1080/09658211.2013.795598 (copyright Taylor \& Francis), available online at: http://www.tandfonline.com/10.1080/09658211.2013.795598

research assistants, who were blind to the original coding independently assigned each event to one of the 34 categories, agreeing on $80 \%$ of all the events. Discrepancies were solved by discussion.

\section{Results}

Of the 34 life story categories, fifteen event categories matched event categories in the CLS of Qatar. Six of these categories (Begin school, Marriage, University/college, Job, Having children and Graduation from university) were among the ten most frequently mentioned categories of life script events. The nineteen new categories primarily regarded travelling, moving, or unexpected events such as accidents and illness. Surprisingly the life story memories of the Qataris showed little religious contents. Only one religious event "Hajj/pilgrimage" is mentioned enough times to be made into a category.

\section{Comparison by gender in Qatar}

To examine gender differences, a series of independent $t$-tests were conducted between the answers from the men and the answers from the women. The only variable that showed a significant difference was "life story typicality". Compared to the women the Qatari men generated more typical life story events, $t(81)=2.773, p=.007, d=.30$, meaning that the men were more likely to nominate high frequency events from the CLS as an important event in their own life story.

\section{The positive life story bump}

Of all life story events mentioned by Qataris, $75 \%$ were dated to be in the bump period. Out of the events remembered from this period, 65\% matched CLS events and most of these events were remembered as positive. In comparison $57 \%$ of all life story events matched the CLS. Since all participants were college students and $48 \%$ of them were aged 20 or younger, the graphs showing life story events were cut off at age 20 . The youngest 
This is an Author's Accepted Manuscript of an article published in Memory (2014), 22:4, 390407, DOI: 10.1080/09658211.2013.795598 (copyright Taylor \& Francis), available online at: http://www.tandfonline.com/10.1080/09658211.2013.795598

participants were aged 17 , so from this age and up to age 20 the graph is corrected for the remaining number of participants. The number of events mentioned for the $18^{\text {th }}$ year was divided by the remaining number of participants and then multiplied by the original number of participants, [(no. of events/remaining no. of participants) x original no. of participants]. The distribution of age estimates for positive, neutral, and negative events (Figure 2) replicates a previous study of Americans in their late teens, with a relative flat beginning and a marked rise of positive events from age fifteen to age eighteen (See Figure 2 in Collins, Pillemer, Ivcevic, \& Gooze, 2007). The positivity bias indicates that this distribution might resemble the standard reminiscence bump for autobiographical memory (Berntsen \& Rubin, 2002) but it is probably at least partly due to a dominance of memories for recent events reflecting ordinary forgetting. Obviously, for young adults, observations of a bump are conflated with such recency effects.

The markedly elevated starting point from the Qatari CLS was not replicated in the life story data. A detailed graph showing the age distribution of CLS events with a two-year interval (Figure 3) reveals that most of the early CLS events are expected to happen before age seven. The fact that these events are little represented among the life story event memories may therefore reflect childhood amnesia. The lowest age for a CLS event in the Qatari life stories was 5.6 years for the event of beginning school and only a total of 4 events were mentioned from before the age of five. These events were: “Accident of my father and he died" age 2 "Before my mom's grandmother died she used to scare me" age 3, and two idiosyncratic events at age four about attending a nursery. None of the participants mentioned events from before the age of 2 . Only three of the life story categories generated up until the age of seven overlapped with the CLS ("Begin School", "Go to school”, "Choosing Friends"). All of these categories are mentioned less frequently in the life stories. 
This is an Author's Accepted Manuscript of an article published in Memory (2014), 22:4, 390407, DOI: 10.1080/09658211.2013.795598 (copyright Taylor \& Francis), available online at: http://www.tandfonline.com/10.1080/09658211.2013.795598

For example, the most frequent event "Begin school" accounts for $28 \%$ of the events estimated to happen before the age of 18 in the CLS and for only $6 \%$ of the events mentioned in life stories (this cut off was chosen because the youngest participant in the life story study was age 17). The event categories from the CLS not mentioned as important life story events all had religious contents except "Ennah" - a traditional hobby activity where men go to the desert.

\section{Discussion}

Study 2 showed that more than half of the life story events matched the CLS event categories, but surprisingly few of these events were religious. In fact only one religious event "Hajj/pilgrimage" was mentioned enough times to become a life story category. The elevated starting point found in the age distribution of positive CLS events was not replicated in the distribution of positive life story events. The lack of both the elevated starting point and religious events in the Qatari life stories supports the interpretation of Study 1 that religious events might account for the dominance of positive events during the first decade in the Qatari CLS. What then makes the religious events less frequently mentioned when the Qataris generate life story events? The most obvious answer might be that the religious events are just not as important when it comes to the personal life story as are educational events such as "Begin school". However, the religious event Hajj, which is estimated to happen around age 20, does appear in the life stories, thereby contradicting this explanation. Similarly, in contrast to this explanation, non-religious CLS events with low age estimates were as infrequent as the religious CLS events. This suggests a more general tendency to leave out events that happen in the first decade of life. For this reason, childhood amnesia might be a better explanation of the failure to replicate the first peak of positive events found between the ages 0-7 in the CLS. 
This is an Author's Accepted Manuscript of an article published in Memory (2014), 22:4, 390407, DOI: 10.1080/09658211.2013.795598 (copyright Taylor \& Francis), available online at: http://www.tandfonline.com/10.1080/09658211.2013.795598

According to studies of childhood amnesia, people recall very little from the first 4-6 years of their life (Nelson, 1993; Pillemer \& White, 1989; Rubin, Wetzler \& Nebes, 1986). A meta study of more than 11,000 autobiographical memories from the first decade of life has shown that the proportion of early childhood memories increases up to the age of seven (Rubin, 2000). More recently, a study by Jack and Hayne (2010) showed a stage-like change in the remission of childhood amnesia. Only sparse and sporadic recall was found before the age of four and, up to age seven, memories were still isolated and infrequent. This is in support of Newcombe et al.'s (2007) proposal of childhood amnesia as a two-stage process, with absolute amnesia in the earliest years and then a period of relative amnesia until adult levels of remembering are in place by age seven. Therefore, events experienced up to age seven can be affected by childhood amnesia. This can explain why the most frequently mentioned event in the Qatari CLS "Begin school” was mentioned almost five times less frequently in the life story memories and why low frequent events estimated to happen early in life (e.g. "Go to the mosque") did not even make the cut off for the life story categories. The absence of earlier religious events such as "Islamic birth rituals" and "Circumcision" may also be attributed to childhood amnesia along with low frequent mentioning of other events happening before the age of four.

To sum up, the considerable overlap found between life story events and CLS events is in partial support of our hypothesis for Study 2. However, since the Qatari life stories hardly showed any gender difference and only very few participants mentioned religious events, no support was found for the part of our hypothesis stating that the specific cultural characteristics of the Qatari CLS would be evident in the life stories. The lack of religious events might partly be due to childhood amnesia. In Study 3, we explore the CLS cross- 
This is an Author's Accepted Manuscript of an article published in Memory (2014), 22:4, 390407, DOI: 10.1080/09658211.2013.795598 (copyright Taylor \& Francis), available online at: http://www.tandfonline.com/10.1080/09658211.2013.795598

culturally by reanalysing the data from Qatar compared to similar data from Denmark, Turkey, and the US.

\section{STUDY 3: Reanalysis and cross-cultural comparison}

Studies 1 and 2 showed a significant overlap between the CLS and life stories of Qatar but some remarkable differences were also detected. The aim of Study 3 was a crosscultural comparison of CLS data from Denmark, Turkey, the US and Qatar. The data from the additional countries were provided from the authors of the original studies (Berntsen and Rubin, 2004; Erdoğan, Baran, Avlar, Çağlar Taş, \& Tekcan, 2008; Rubin, Berntsen, \& Hutson, 2009). In the present study, they are reanalysed to see if the Qatari CLS differ compared to life scripts from more secular societies when using the same variables as used in Study 1.

\section{Age distributions}

Consistency was found between the four countries with regard to the bump for positive events expected to happen between the ages 15-30 (Figure 4) and the similarity between the four bump curves was enhanced when removing all religious events from the four datasets causing the elevated starting point for Qatar to disappear. The plot for the positive Turkish events also flattened slightly in the first decade when controlling for religious content (Figure 4). Across all four samples more than half of all CLS events were estimated to occur within the short period of the 15 years that make the bump period.

\section{Cross-cultural comparison of the CLS}

Table 5 gives an overview of the CLS from the four countries. The CLS of Qatar includes sixteen event categories with content specifically found in Qatar and not in the CLS of the other three countries examined. In comparison, the Danish CLS has eight events 
This is an Author's Accepted Manuscript of an article published in Memory (2014), 22:4, 390407, DOI: 10.1080/09658211.2013.795598 (copyright Taylor \& Francis), available online at: http://www.tandfonline.com/10.1080/09658211.2013.795598

specific to their own country, the Turkish CLS seven, and the American CLS only one (Table 5). In the Qatari CLS, eight of the country specific event categories are religious and $25 \%$ of all categories have religious content. This shows a high focus on religiosity compared to Denmark with 6\% religious categories (confirmation and baptism) and Turkey with $4 \%$ religious categories (circumcision - only mentioned by males). The Americans generated no religious categories. The religious events in the CLS from the Muslim countries were mainly generated by men. This was not the case in the Danish CLS. As for the events estimated to happen before the age of seven, $31 \%$ of the events generated by the Qataris had religious content, while this was only the case for $11 \%$ of the Turkish events and $4 \%$ of the Danish events. Counting categories with a mean for emotional valence below -.50, the Turkish CLS is the least positive with $37 \%$ of their event categories being classified as negative. The Danish CLS shows $23 \%$ of the categories to be negative and the US $17 \%$ (Table 5). In comparison, the Qatari CLS has only 8\% negative categories.

To further examine the differences across cultures, a series of one way ANOVAs were conducted using the dependent variables from Study 1 (Table 1). A main effect of country was found for all six dependent variables (Table 6). Somewhat surprisingly the post hoc tests showed that the Qataris generated relatively many “other" events - that is, events that could not be classified according to a life script category. The frequency of such "idiosyncratic events" in the Qatari CLS was not significantly different from the American CLS, which was characterised by a high number of idiosyncratic events (Rubin et al., 2009). The post hoc test confirmed that, compared to the three other countries, the Qataris focused more on "country specific events", "religious events", events with low "age estimates" and events with a positive "script valence" (all $p$ 's $<.001$ - see Table 6 for superscripts regarding the post hoc test). The Qataris and the Americans both showed life expectations in 
This is an Author's Accepted Manuscript of an article published in Memory (2014), 22:4, 390407, DOI: 10.1080/09658211.2013.795598 (copyright Taylor \& Francis), available online at: http://www.tandfonline.com/10.1080/09658211.2013.795598

the CLS that were significantly more positive than the Danish and Turkish (all $p$ 's $<.001-$ see Table 6). A follow up t-test between the CLS of Qatar and the US showed a trend towards the Qataris having the most positive expectations, $t(149)=1.916, p=.057, d=.16$. Again the cause might be found in the categories unique to Qatar, which contain many positive religious events that are estimated to happen at a young age.

\section{Summary and Discussion}

Compared to the other countries the Qataris had the most positive CLS, which was characterised by country specific and religious events happening early in life. When controlling for religious content, the frequency plot for estimated positive events flattened remarkably in the first decade of a normative Qatari life. Without religious events, the distribution of positive events from Qatar resembles the curves from Denmark, Turkey, and the US (Figure 4). This shows that the cultural expectation of positive religious events is creating the elevated starting point found in the distribution of age estimates for positive events in the Qatari CLS. The CLS of the secular Muslim country in the present Study showed a similar but less evident pattern. The plot for the positive Turkish events flattened slightly and like in Qatar the religious events were mainly generated by the men. This gender difference was probably influenced by the fact, that the only religious event category generated by the Turks was for the male event "circumcision". As for the multi-religious country, the US, no particular religious events were mentioned enough times to be made into a category in the CLS. It is possible that Americans may view religion as an individual choice that is too diverse to be culturally scripted. The non-religious, but traditionally Protestant country of Denmark, showed two religious categories in the CLS. However, in Denmark the mentioned events of baptism and confirmation are likely to be viewed more as a tribute to tradition rather than as an act of religion (Zuckerman, 2008). 
This is an Author's Accepted Manuscript of an article published in Memory (2014), 22:4, 390407, DOI: 10.1080/09658211.2013.795598 (copyright Taylor \& Francis), available online at: http://www.tandfonline.com/10.1080/09658211.2013.795598

Despite the differences found with regard to script valence and in the religious focus, Study 3 showed many similarities between the CLS of Qatar, Turkey, Denmark, and the US. The most notable being the dominance of positive events estimated to happen in young adulthood. The distribution of these events follows the standard reminiscence bump for autobiographical memory (Berntsen \& Rubin, 2002).

\section{General Discussion}

Through 3 studies, we have examined the CLS of Qatar. Comparisons have not only been made to the Qataris' own life story events but also to the CLS of three countries differing from Qatar in various ways. The resemblance across cultures was striking but some notable differences were also found.

The clearest difference in the Qatari CLS compared to the CLS from Turkey, Denmark, and the US was the focus on religious events. The dominance of religious events is consistent with findings in the Muslim sample from Malaysia even though a different method was used to obtain this CLS (Haque \& Hasking, 2010). Religious content was found in approximately one third of the CLS events expected by the Qataris to happen before the age of seven and this marked focus on religious events accounted for the dominance of positive events in the first decade event categories. Qataris showed strong agreement about the timing of religious events and of positive events in general, consistent with previous studies (Berntsen \& Rubin, 2004; Bohn, 2010; Erdoğan et al., 2008; Rubin et al., 2009). The fact that a non-secular country such as Qatar generated more religious event categories than what is previously found in a CLS supports the hypothesis stating that cultural norms structure expectations to the content and order of events in a typical life course (Berntsen \& Rubin, 2004; Habermas \& Bluck, 2000). 
This is an Author's Accepted Manuscript of an article published in Memory (2014), 22:4, 390407, DOI: 10.1080/09658211.2013.795598 (copyright Taylor \& Francis), available online at: http://www.tandfonline.com/10.1080/09658211.2013.795598

The gender differences in the Qatari CLS were mainly found between-subjects. Qatari men generated more events specific to Qatar, including religious events happening at a young age, while women generated more cross-cultural events. This gender different focus in nominating life events to the CLS portrays Qatar as a segregated society with two gender specific subcultures that differ in their expectations towards a normative life. A male subculture with a culturally specific religious CLS and a female subculture with a more cross-cultural CLS. This is in accordance with the suggestion that a CLS may vary across groups within a country (Haque \& Hasking, 2010; Collins et al., 2007).

What then makes the Qatari men more prone to mentioning events specific to their own culture? Could there be a gender difference with regard to the preservation of culture in societies where children are raised to value gender role segregation? Baumeister (2010) regards culture as a system of explicit and tacit rules that enables a human group to work together effectively. He argues that there are gender differences in the motivation for social interaction. Women tend to create close intimate relationships while men rather socialise in larger networks of shallower relationships. The intimate relationships are crucial for the survival of the human species while the large social networks are the foundation of culture (Baumeister, 2010). If men are more motivated for building large networks, thereby creating culture, they might also have a more explicit focus on maintaining culture. This is in agreement with a study by Harris (1994) who found that men perceive the role as standardbearers to be one of the most positive male roles. The essence of this role is to pass on cultural traditions and moral values to the next generation. Because traditional rituals serve as a tool for passing on knowledge from one generation to the next, the role of standardbearer might be more evident in societies with many religious events and rituals (Geertz, 1999). 
This is an Author's Accepted Manuscript of an article published in Memory (2014), 22:4, 390407, DOI: 10.1080/09658211.2013.795598 (copyright Taylor \& Francis), available online at: http://www.tandfonline.com/10.1080/09658211.2013.795598

The only difference found within-subjects with regard to the CLS generated for the male versus the female role also concerned religious events. Both men and women mentioned religious events to a higher extent for the male role, although this effect was most pronounced among the male participants. A possible explanation as to why religious event categories were the most evident in the male CLS might be that Muslim men are expected to practice their religion more explicitly than women by going to mosques and attending public Islamic events (El Guindi, 2003). The questionnaire in the present study specifically asked about events and, therefore, it might not relate as well to the religious script for Qatari women who mostly practise religion at home as an integrated and uneventful part of everyday life. This is in accordance with Loewenthal et al.'s (2002) study of religious traditions. They found that Muslim, Hindu and Jewish women show less explicit religious activity than men from the same religions without necessarily being less cognitively or emotionally religious.

Hardly any gender differences were found in the remembered life story events. Even more surprisingly, the life story memories of the Qataris showed little religious content at all. The fact that religious events are mentioned in the Qatari CLS but not in the life stories suggests that these young participants emphasise religion as a cultural model for life as a Muslim, rather than a model made of their own personal memories (Geertz, 1966). Even though they might lack memory of these events, their parents probably made sure that they followed this religious model depicted in the CLS. Almost all the religious event categories in the Qatari CLS were expected to occur early in life and, therefore, childhood amnesia may at least partly explain why these events were not reported as important life story memories - that is, they are simply not remembered by the person as personal experiences. Interestingly, even with this lack of religious events, a considerable overlap was still found 
This is an Author's Accepted Manuscript of an article published in Memory (2014), 22:4, 390407, DOI: 10.1080/09658211.2013.795598 (copyright Taylor \& Francis), available online at: http://www.tandfonline.com/10.1080/09658211.2013.795598

between the Qataris' life story events and the CLS of Qatar. Out of all life story events, 57\% matched the Qatari CLS. In the US 46\% events matched and in Denmark 70\% events matched (Rubin et al., 2009). In general, these overlaps support previous findings suggesting that knowledge of a normative life affect the memory of important life story events (Bohn \& Berntsen, 2008; Glück \& Bluck, 2007; Rubin \& Berntsen, 2003; Thomsen \& Berntsen, 2008).

Differences were found with regard to religiousness, emotional valence, and gender, but the CLS of Qatar still showed characteristics similar to the CLS of other countries (Berntsen \& Rubin, 2004; Erdoğan et al., 2008; Habermas, 2007; Haque \& Hasking, 2010; Janssen \& Rubin, 2011; Rubin et al., 2009). The overlap of event categories is remarkable taking into consideration that Qatar is a country less WEIRD than those previously explored. Most of the high frequent categories have the same content across cultures and, in accordance with previous findings, the Qataris showed strong agreement on the age estimates of positive events in young adulthood. This positivity bias combined with a marked focus on expected events between the ages of 15-30 is in support of the CLS account of the reminiscence bump stating that people across cultures use the CLS for guiding the retrieval of autobiographical memories (Berntsen \& Rubin, 2004).

The present study has a number of limitations. We chose university students as participants partly because the Qatari society is a relatively closed society with limited access to native Qataris and partly because it made the present studies comparable with previous studies, which likewise used college students. While this choice was well motivated for the sake of cross-cultural comparison, limited diversity with regard to age, educational level, and wealth might make these samples less representative of the general populations. The age, however, appears to have little effect on the CLS, according to 
This is an Author's Accepted Manuscript of an article published in Memory (2014), 22:4, 390407, DOI: 10.1080/09658211.2013.795598 (copyright Taylor \& Francis), available online at: http://www.tandfonline.com/10.1080/09658211.2013.795598

findings showing that people across ages generate similar life scripts (Bohn, 2010; Janssen \& Rubin, 2011). The same applies to levels of education, according to Janssen and Rubin's (2011) study of the CLS in the Netherlands. Consistent with previous studies, we used a 4\% cut-off point when scoring for the event categories in the Qatar CLS. This leaves open the possibility that the least frequently mentioned events portray idiosyncrasy instead of culturally shared knowledge. However, given the open-ended instructions and the fact that the participants were limited to generating only 7 events that they deemed most important in a prototypical life course, we have reasons to believe that when events match across participants it is likely to be culturally shared knowledge at least to some extent. In order not to contaminate the CLS of Qatar (which, following previous studies, is built on each participant generating events for their own gender), we had all the participants answer for their own gender first. This lack of counter balancing might have influenced the results. The participants may have felt an urge to provide different events for the other gender. However, this is not likely since very little difference was found within-subjects regarding the male versus the female role. In general, the male participants had different expectations to a normative Qatari life compared to the female participants, but each participant did not differ significantly between the male and female roles in their answers.

Despite these limitations the present studies have contributed with new knowledge regarding the CLS. The examination of the Qatari CLS showed clear effects of gender and religion by suggesting the presence of two gender specific subcultures with different expectations towards a normative life. The subcultures can be described as a male subculture that focused on cultural events specific to Qatar including religious events and a female subculture more focused on cross-cultural events. These effects were not evident in the Qatari life story events. Future studies should clarify whether or not this lack of gender 
This is an Author's Accepted Manuscript of an article published in Memory (2014), 22:4, 390407, DOI: 10.1080/09658211.2013.795598 (copyright Taylor \& Francis), available online at: http://www.tandfonline.com/10.1080/09658211.2013.795598

specific and religious events in the life stories is due to the young age of the participants or other potential factors, such as level of education. However, a considerable overlap of events was still found between the life stories and the CLS of Qatar, supporting the guiding function of the CLS in retrieval of autobiographical memories. 
This is an Author's Accepted Manuscript of an article published in Memory (2014), 22:4, 390407, DOI: 10.1080/09658211.2013.795598 (copyright Taylor \& Francis), available online at: http://www.tandfonline.com/10.1080/09658211.2013.795598

\section{Acknowledgements}

This work was supported by the Danish National Research Foundation (DNRF93) and by the MindLab UNIK initiative at Aarhus University, which is funded by the Danish Ministry of Science and Technology and Innovation. In addition we would like to thank Ali I. Tekcan and David C. Rubin for granting access to data from the original studies of the CLS from Turkey and the US. We would also like to thank Fadwa El Guindi, Ali Al Shawi, Paul Sillitoe, Salah Al Mannai and Azza Elnayal for help with recruiting participants. Najla Majid, Sine Scanlon, and Thea Ejby Salmonsen are thanked for helping with the scoring of the data. Finally, our thanks go to Jonathan Koppel, Annette Bohn, Kim Berg Johannessen, and David Pillemer for their helpful suggestions and comments in the process. 
This is an Author's Accepted Manuscript of an article published in Memory (2014), 22:4, 390407, DOI: 10.1080/09658211.2013.795598 (copyright Taylor \& Francis), available online at: http://www.tandfonline.com/10.1080/09658211.2013.795598

\section{References}

Bahry, L., \& Marr, P. (2005). Qatari women: A new generation of leaders? Middle East Policy. 12(2), 104-119.

Baumeister, R. F. (2010). Is there anything good about men? How cultures flourish by exploiting men. New York NY: Oxford University Press.

Berntsen, D., \& Rubin, D. C. (2002). Emotionally charged autobiographical memories across the life span: The recall of happy, sad, traumatic, and involuntary memories. Psychology and Aging. 17(4), 636-652.

Berntsen, D., \& Rubin, D. C. (2004). Cultural life scripts structure recall from autobiographical memory. Memory \& Cognition. 32(3), 427-442.

Blackwood, E. (2006). Women, Islam and modernity: Single women, sexuality and reproductive health in contemporary Indonesia. United Kingdom: Sage Publications, London UK.

Bluck, S., \& Habermas, T. (2000). The life story schema. Motivation and Emotion. 24(2), 121-147.

Bohn, A., \& Berntsen, D. (2008). Life story development in childhood: The development of life story abilities and the acquisition of cultural life scripts across late middle childhood and adolescence. Developmental Psychology, 44, 1135-1147.

Bohn, A. (2010). Generational differences in cultural life scripts and life story memories of younger and older adults. Applied Cognitive Psychology. 24(9), 1324-1345. 
This is an Author's Accepted Manuscript of an article published in Memory (2014), 22:4, 390407, DOI: 10.1080/09658211.2013.795598 (copyright Taylor \& Francis), available online at: http://www.tandfonline.com/10.1080/09658211.2013.795598

Bohn, A., \& Berntsen, D. (2011). The reminiscence bump reconsidered: Children's prospective life stories show a bump in young adulthood. Psychological Science. 22(2), 197-202.

Clinton, M. (2011). Mental health reform in Qatar. International Journal of Mental Health Nursing. 20(5), 308-309.

Collins, K. A., Pillemer, D. B., Ivcevic, Z., \& Gooze, R. A. (2007). Cultural scripts guide recall of intensely positive life events. Memory \& Cognition. 35(4), 651-659.

El Guindi, F. (2003). Veil: modesty, privacy and resistance (No. 10). Berg Publishers.

Erdoğan, A., Baran, B., Avlar, B., Taş, A. Ç, \& Tekcan, A. İ. (2008). On the persistence of positive events in life scripts. Applied Cognitive Psychology. 22(1), 95-111.

Geertz, A. W. (1999). Definition as analytical strategy in the study of religion. Historical Reflections. 25(3), 445-453.

Geertz, C., \& Banton, M. (1966). Religion as a cultural system (pp. 1-46). London: Tavistock.

Glück, J., \& Bluck, S. (2007). Looking back across the life span: A life story account of the reminiscence bump. Memory \& cognition, 35(8), 1928-1939.

Habermas, T., (2007). How to tell a life: The development of the cultural concept of biography. Journal of Cognition and Development. 8(1), 1-31.

Habermas, T., \& Bluck, S. (2000). Getting a life: The emergence of the life story in adolescence. Psychological Bulletin. 126(5), 748-769. 
This is an Author's Accepted Manuscript of an article published in Memory (2014), 22:4, 390407, DOI: 10.1080/09658211.2013.795598 (copyright Taylor \& Francis), available online at: http://www.tandfonline.com/10.1080/09658211.2013.795598

Hamzeh, A. N. (1994). Qatar: The duality of the legal system. Middle Eastern Studies. 30(1), 79-90.

Haque, S., \& Hasking, P. A. (2010). Life scripts for emotionally charged autobiographical memories: A cultural explanation of the reminiscence bump. Memory. 18(7), 712-729.

Harris, I. M. (1994). Men as standard bearers. The Journal of Men's Studies. 3(2), 103-125.

Henrich, J., Heine, S. J., \& Norenzayan, A. (2010). The weirdest people in the world? Behavioral and Brain Sciences. 33(2-3), 61-135.

Hulsether, M. (2005). Religion and Culture. The Routledge Companion to the Study of Religion, 489-508.

Jack, F., \& Hayne, H. (2010). Childhood amnesia: Empirical evidence for a two-stage phenomenon. Memory. 18(8), 831-844.

Janssen, S. M. J., \& Rubin, D. C. (2011). Age effects in cultural life scripts. Applied Cognitive Psychology. 25(2), 291-298.

Loewenthal, K. M., MacLeod, A. K., \& Cinnirella, M. (2002). Are women more religious than men? Gender differences in religious activity among different religious groups in the UK. Personality and Individual Differences. 32(1), 133-139.

Looney, R. E. (1992). Effect of economic environment on Arab industrialisation. Middle East Business and Economic Review, 4(1), 26-40. 
This is an Author's Accepted Manuscript of an article published in Memory (2014), 22:4, 390407, DOI: 10.1080/09658211.2013.795598 (copyright Taylor \& Francis), available online at: http://www.tandfonline.com/10.1080/09658211.2013.795598

McAdams, D. P. (1993). The stories we live by: Personal myths and the making of the self. New York, NY, US: William Morrow \& Co.

McAdams, D. P. (1996). Personality, modernity, and the storied self: A contemporary framework for studying persons. Psychological Inquiry. 7(4), 295-321.

Nelson, K. (1993). Explaining the emergence of autobiographical memory in early childhood. In A. F. Collins, S. E. Gathercole, M. A. Conway \& P. E. Morris (Eds.), Theories of memory. Hillsdale, NJ, England: Lawrence Erlbaum Associates, Inc. pp. 355-385

Neugarten, B. L., Moore, J. W., \& Lowe, J. C. (1965). Age norms, age constraints and adult socialization. American Journal of Sociology. 70(6), 710-717.

Neugarten, B. L. (1968). Adult personality: Toward a psychology of the life cycle. In B. L. Neugarten (Ed.), Middle age and aging: A reader in social psychology (pp. 137-147). Chicago: University of Chicago Press.

Newcombe, N. S., Lloyd, M. E., \& Ratliff, K. R. (2007). Development of episodic and autobiographical memory: A cognitive neuroscience perspective. In R. V. Kail (Ed.), Advances in Child Development and Behavior. 35, 37-85.

Pillemer, D., \& White, S. H. (1989). Childhood events recalled by children and adults. In H. W. Reese (ed.), Advances in Child Development and Behavior. 21, 297-340

Qatar general secretariat for development planning (2011). Qatar national development strategy 2011-2016. Doha: Gulf Publishing and Printing Company. 
This is an Author's Accepted Manuscript of an article published in Memory (2014), 22:4, 390407, DOI: 10.1080/09658211.2013.795598 (copyright Taylor \& Francis), available online at: http://www.tandfonline.com/10.1080/09658211.2013.795598

Rathmell, A. (2000). Political reform in the Gulf: The case of Qatar. Middle Eastern Studies, $36(4), 47-62$.

Rubin, D. C., \& Kontis, T. C. (1983). A schema for common cents. Memory \& Cognition, 11, $335-341$.

Rubin, D. C., Stolzfus, E. R., \& Wall, K. L. (1991). The abstraction of form in semantic categories. Memory \& Cognition, 19, 1-7.

Rubin, D. C. (2000). The distribution of early childhood memories. Memory. 8(4), 265-269.

Rubin, D. C., \& Berntsen, D. (2003). Life scripts help to maintain autobiographical memories of highly positive, but not highly negative, events. Memory \& Cognition. 31(1), 1-14.

Rubin, D. C., Berntsen, D., \& Hutson, M. (2009). The normative and the personal life: Individual differences in life scripts and life story events among USA and Danish undergraduates. Memory. 17(1), 54-68.

Rubin, D. C., Wetzler, S. E., \& Nebes, R. D. (1986). Autobiographical memory across the lifespan. In D. C. Rubin (Ed.), Autobiographical memory (pp. 202-221). New York, NY, US: Cambridge University Press.

Schank, R. C., \& Abelson, R. P. (1977). Scripts, plans, goals and understanding: An inquiry into human knowledge structures. Oxford, England: Lawrence Erlbaum.

Shweder, R.A. (1998). Welcome to middle age! : (and other cultural fictions). Chicago, University of Chicago Press. 
This is an Author's Accepted Manuscript of an article published in Memory (2014), 22:4, 390407, DOI: 10.1080/09658211.2013.795598 (copyright Taylor \& Francis), available online at: http://www.tandfonline.com/10.1080/09658211.2013.795598

Taras, V., Kirkman, B. L., \& Steel, P. (2010). Examining the impact of Culture's consequences: A three-decade, multilevel, meta-analytic review of Hofstede's cultural value dimensions. Journal of Applied Psychology, 95(3), 405.

Thomsen, D.K., \& Berntsen, D. (2008). The cultural life script and life story chapters contribute to the reminiscence bump. Memory, 16, 420-435.

Zuckerman, P. (2008). Society without god: What the least religious nations can tell us about contentment. New York, NY: New York University Press. 
This is an Author's Accepted Manuscript of an article published in Memory (2014), 22:4, 390407, DOI: 10.1080/09658211.2013.795598 (copyright Taylor \& Francis), available online at: http://www.tandfonline.com/10.1080/09658211.2013.795598

\section{Footnotes}

${ }^{1}$ In Qatar, menarche is not just a biological manifestation of a girl becoming a woman. From this day on veiling is proscribed by the prophet Muhammad. During a woman's period, she is excused from religious duties such as praying and visiting religious grounds. Likewise, during Ramadan she is excused from fasting, but it is expected that she will catch up by fasting at a more convenient time (Blackwood, 2006).

2 "Gender divided social skills" regard gender differences in dress code, public appearance, and domestic responsibilities. 
This is an Author's Accepted Manuscript of an article published in Memory (2014), 22:4, 390407, DOI: 10.1080/09658211.2013.795598 (copyright Taylor \& Francis), available online at: http://www.tandfonline.com/10.1080/09658211.2013.795598

\section{Table 1}

Definitions of the dependent variables used to explore differences across gender and country

Script valence

Idiosyncratic events

Religious events

Cross-cultural events

Country specific events

Age Estimate*

Life story typicality*

Story valence*
A mean of the valence ratings given by each participant to CLS events.

Sum of events in a participant's CLS that are classified as "other" in the overall CLS .

Sum of events in a participant's CLS that are classified as "religious" in their own country's CLS.

Sum of events in a participant's CLS that are present in all 4 CLS and thus shared across culture (events classified as "other" are not counted).

Sum of events in a participant's CLS that are only present in and therefore unique to the CLS of their own culture.

The average of the age estimate given by each participant to events in their CLS. A measure of age norms.

The mean score of CLS frequency norms** for each participant's seven life story events. Calculated in percentages for comparison across countries.

A mean of the valence ratings given by each participant to life story events.

* "Age estimates" only concerns study 1, whereas "story valence" and "typicality" concern study 2 .

** Frequency scores taken from Hutson et al. (2009, table 2) for the American participants; Berntsen and Rubin (2004, Table 3) for the Danish participants; Erdoğan et al. (2008, table 1) for the Turkish participants; and in the present study's Table 2 for the Qatari participants. 
This is an Author's Accepted Manuscript of an article published in Memory (2014), 22:4, 390-

407, DOI: 10.1080/09658211.2013.795598 (copyright Taylor \& Francis), available online at:

http://www.tandfonline.com/10.1080/09658211.2013.795598

\section{Table 2}

The cultural life script of Qatar ranked by of participants mentioning the same event.

\begin{tabular}{|c|c|c|c|c|c|c|c|c|c|}
\hline & & & \multirow{2}{*}{\multicolumn{2}{|c|}{ Event }} & \multirow{2}{*}{$\frac{\text { Records }}{\frac{\circ}{\circ}}$} & \multirow{2}{*}{$\frac{\text { Age a }}{\mathrm{M}}$} & \multirow{2}{*}{$\frac{\text { at event }}{S D}$} & \multicolumn{2}{|c|}{ Emotion } \\
\hline & & & & & & & & $\overline{\mathrm{M}}$ & $\mathrm{SD}$ \\
\hline & & $\mathrm{CC}$ & 01. & Begin school & 82 & 6.85 & 2.05 & 1.98 & 1.49 \\
\hline & & $\mathrm{CC}$ & 02. & Marriage & 64 & 23.51 & 3.08 & 2.50 & 0.96 \\
\hline & & $\mathrm{CC}$ & 03. & University/College & 49 & 19.22 & 1.55 & 2.31 & 1.26 \\
\hline & & $\mathrm{CC}$ & 04. & Job & 31 & 22.80 & 1.86 & 2.24 & 1.60 \\
\hline & & $\mathrm{CC}$ & 05. & Kindergarten/Day Care & 27 & 4.36 & 1.08 & 0.57 & 2.24 \\
\hline & & $\mathrm{Cc}$ & 06. & Puberty & 24 & 13.83 & 1.40 & 1.50 & 2.07 \\
\hline & & $\mathrm{CC}$ & 07. & Having Children & 24 & 24.92 & 3.68 & 2.58 & 0.67 \\
\hline \multirow[t]{3}{*}{ Q } & & & 08. & Graduation from university & 22 & 23.00 & 1.28 & 2.92 & 0.29 \\
\hline & & & 09. & Own Birth & 20 & 0.10 & 0.32 & 1.90 & 2.13 \\
\hline & & & 10. & Begin Driving & 20 & 19.18 & 1.99 & 2.00 & 1.79 \\
\hline \multirow[t]{3}{*}{ Q } & & & 11. & Graduation Secondary School & 20 & 17.60 & 1.08 & 2.27 & 1.85 \\
\hline & $\mathrm{R}$ & & 12. & Circumcision & 18 & 0.33 & 0.50 & 2.50 & 1.27 \\
\hline & & & 13. & Choosing friends & 16 & 11.22 & 3.46 & 0.67 & 1.50 \\
\hline Q & & & 14. & Gender divided social skills & 15 & 7.67 & 4.59 & 2.63 & 0.74 \\
\hline Q & $\mathrm{R}$ & & 15. & Learn Islamic value \& moral & 11 & 5.20 & 1.30 & 2.60 & 0.89 \\
\hline Q & & & 16. & Raising children & 9 & 26.80 & 1.92 & 1.20 & 1.79 \\
\hline \multirow[t]{3}{*}{ Q } & $\mathrm{R}$ & & 17. & Islamic birth rituals & 7 & 0.00 & 0.00 & 2.25 & 0.96 \\
\hline & & $\mathrm{CC}$ & 18. & Begin walking & 7 & 0.50 & 0.71 & 2.75 & 0.50 \\
\hline & & $\mathrm{Cc}$ & 19. & Go to school & 7 & 15.00 & 1.41 & 1.75 & 1.26 \\
\hline \multirow[t]{4}{*}{ Q } & $\mathrm{R}$ & & 20. & Learning to pray & 7 & 7.00 & 1.63 & 2.25 & 1.50 \\
\hline & & & 21. & Major achievement & 7 & 16.75 & 4.79 & 2.75 & 0.50 \\
\hline & & $\mathrm{CC}$ & 22. & Own death & 7 & 60.00 & - & -2.25 & 1.50 \\
\hline & & $\mathrm{CC}$ & 23. & Growing old/Retirement & 7 & 53.75 & 4.79 & 0.00 & 3.00 \\
\hline Q & & & 24. & Women's independence & 7 & 45.25 & 4.11 & 1.25 & 2.36 \\
\hline \multirow[t]{3}{*}{ Q } & $\mathrm{R}$ & & 25. & Go to the mosque & 7 & 6.75 & 1.26 & 3.00 & 0.00 \\
\hline & & $\mathrm{CC}$ & 26. & Begin talking & 7 & 2.33 & 2.36 & 2.75 & 0.50 \\
\hline & & $\mathrm{CC}$ & 27. & Death of relative/others & 7 & 31.50 & 26.16 & -2.25 & 0.96 \\
\hline \multirow[t]{2}{*}{ Q } & & & 28. & Parents help with settling & 5 & 28.33 & 10.41 & 2.33 & 0.58 \\
\hline & & & 29. & Travel \& studying abroad & 5 & 18.67 & 8.08 & 2.00 & 1.00 \\
\hline \multirow[t]{2}{*}{$Q$} & & & 30. & Stability in life & 5 & 31.33 & 8.08 & 1.00 & 3.46 \\
\hline & & & & Moving & 4 & 24.00 & - & 2.50 & 0.71 \\
\hline Q & $\mathrm{R}$ & & 32 . & Learn fasting & 4 & 9.00 & 0.00 & 1.00 & 2.83 \\
\hline Q & $\mathrm{R}$ & & 33. & HAJJ - pilgrim fair & 4 & 27.00 & 4.24 & 3.00 & 0.00 \\
\hline Q & $\mathrm{R}$ & & & Learning the Quran & 4 & 1.00 & - & 2.50 & 0.71 \\
\hline Q & & & 35 . & Ennah (trip for young men) & 4 & 26.00 & 12.73 & 2.00 & 0.00 \\
\hline \multirow{2}{*}{ Q } & $\mathrm{R}$ & & 36. & Menarche & 4 & 12.00 & 0.00 & -1.00 & 2.83 \\
\hline & & & 37. & Other & 80 & 15.37 & 10.89 & 1.79 & 1.84 \\
\hline
\end{tabular}

$\mathbf{Q}=$ Unique event categories for Qatar

$\mathbf{R}=$ Religious event categories

$\mathbf{C c}=$ Cross-cultural event categories found in all 4 CLS 
This is an Author's Accepted Manuscript of an article published in Memory (2014), 22:4, 390-407, DOI: 10.1080/09658211.2013.795598 (copyright

Taylor \& Francis), available online at: http://www.tandfonline.com/10.1080/09658211.2013.795598

\section{Table 3}

Gender comparison of CLS events mentioned by at least 3 participants and ranked by of participants mentioning the same event. Religious event categories marked in BOLD.

\section{Female-Life}

\section{Women's answers}

Event categories

1. Begin school

2. Marriage

3. University/College

4. Kindergarten/Daycare

5. Having Children

6. Job

7. Puberty

8. Choosing friends

9. Graduation university

10. Own Birth

11. Graduation 2. School

12. Begin driving

13. Raising children

14. Major achievement

15. Women's independence

16. Gender div. social skill

17. Secondary school

18. Growing old

19. Begin talking
Men's answers

\% Event categories

Marriage

Begin school

Own birth

Gender div. social skill

University/College

Having Children

Graduation High School

Circumcision*

Graduation University

Choosing friends

Islamic birth rituals

Learning to pray

Raising children

Women's independence

\section{Male-Iife}

\section{Women's answers}

Event categories

Begin school

Marriage

University/College

Job

Begin driving

Kindergarten/Daycare

Puberty

Graduation 2. School

Having Children

Own birth

Begin walking

Secondary school

Islamic birth rituals

Major achievement

Own death

Growing old

Death of parent
Men's answers

Event categories

100 Begin school 71

74 Marriage

55 Circumcision

52 Job

42 University/College

32 Begin driving

29 Graduation University

26 Own birth

23 Graduation High School

16 Kindergarten/Daycare

13 Gender div. social skills

13 Learn Islamic value/moral

10 Puberty

10 Islamic birth rituals

10 Go to the mosque

10 Having children

10 Begin walking

Learning to pray

Travel \& study abroad

* Circumcision of girls is not a Qatari custom. The answers in the present study might be due to the Sudanese minority in Qatar. 
This is an Author's Accepted Manuscript of an article published in Memory (2014), 22:4, 390-407, DOI: 10.1080/09658211.2013.795598 (copyright Taylor \& Francis), available online at: http://www.tandfonline.com/10.1080/09658211.2013.795598

\section{Table 4}

Repeated measures ANOVAs showing the CLS characteristics of male role versus female role generated by men versus generated by women.

\begin{tabular}{|c|c|c|c|c|c|c|c|c|c|c|c|c|c|}
\hline \multicolumn{2}{|c|}{ Female } & \multicolumn{2}{|l|}{ CLS } & \multicolumn{4}{|c|}{ Male CLS } & \multicolumn{4}{|c|}{ Main effects } & & \\
\hline \multirow[b]{2}{*}{ Women's $F$} & \multirow[b]{2}{*}{ Answers } & \multirow{2}{*}{\multicolumn{2}{|c|}{ Men's Answers }} & \multirow{3}{*}{$\frac{\text { Women's }}{\mathrm{M}}$} & \multirow[b]{2}{*}{ Answers } & \multirow[b]{2}{*}{$\underline{\text { Men's }}$} & \multirow[b]{2}{*}{ Answers } & \multicolumn{2}{|l|}{$\underline{\mathrm{CLS}}$} & \multicolumn{2}{|c|}{ Answers from } & \multirow{2}{*}{\multicolumn{2}{|c|}{ Interact }} \\
\hline & & & & & & & & Female vs. & Male & Women $v$ & s. Men & & \\
\hline $\mathrm{M}$ & $\overline{\mathrm{SD}}$ & $\mathrm{M}$ & $\overline{\mathrm{SD}}$ & & $S D$ & M & $\mathrm{SD}$ & $F$ & $\overline{\eta_{p}{ }^{2}}$ & $F$ & $\eta_{p}^{2}$ & $\mathrm{~F}$ & $\eta_{p}^{2}$ \\
\hline 15.25 & 6.98 & 12.54 & 5.57 & 17.17 & 6.13 & 13.19 & 3.91 & 3.16 & 0.06 & $4.63^{*}$ & 0.09 & 0.78 & 0.02 \\
\hline 1.72 & 0.97 & 1.80 & 0.76 & 1.39 & 1.14 & 1.88 & 0.84 & 0.61 & 0.01 & 1.66 & 0.03 & 1.72 & 0.04 \\
\hline 0.16 & 0.58 & 0.83 & 1.09 & 0.23 & 0.50 & 1.29 & 1.00 & $8.98^{* *}$ & 0.15 & $19.00^{* \star *}$ & 0.26 & $5.09^{*}$ & 0.09 \\
\hline 0.68 & 0.79 & 1.62 & 2.32 & 0.87 & 1.09 & 1.13 & 1.04 & 0.48 & 0.01 & 3.92 & 0.07 & 2.45 & 0.04 \\
\hline 1.00 & 1.10 & 1.87 & 1.30 & 0.74 & 0.82 & 1.79 & 1.14 & 1.35 & 0.03 & $14.18^{* * *}$ & 0.21 & 0.35 & 0.01 \\
\hline 4.32 & 1.36 & 2.30 & 1.43 & 4.25 & 1.56 & 2.87 & 1.42 & 2.05 & 0.40 & $21.50^{* * *}$ & 0.31 & 3.41 & 0.07 \\
\hline
\end{tabular}

Note: df range $(1,47-53), * p<0.05, * * p<0.01, * * * p<0.001$ 
This is an Author's Accepted Manuscript of an article published in Memory (2014), 22:4, 390-407, DOI: 10.1080/09658211.2013.795598 (copyright

Taylor \& Francis), available online at: http://www.tandfonline.com/10.1080/09658211.2013.795598

\section{Table 5}

Comparison of CLS events across countries ranked by the frequency of participants mentioning the same event. cross-cultural event categories are marked in bold and the mean emotional valence is displayed to the right.

\begin{tabular}{|c|c|c|c|c|c|c|c|c|}
\hline & QATAR & $\mathbf{M}_{\text {emo }}$ & DENMARK & $\mathbf{M}_{\mathrm{emo}}$ & TURKEY & $\mathbf{M}_{\mathrm{emo}}$ & USA & $\mathbf{M}_{\text {emo }}$ \\
\hline 1 . & Begin school & $\overline{2.0}$ & Having Children & $\overline{2.6}$ & Marriage & $\overline{2.0}$ & Marriage & $\overline{2.7}$ \\
\hline 2 . & Marriage & 2.5 & Marriage & 2.5 & Begin School & 1.8 & Having Children & 2.7 \\
\hline 3 . & University/College & 2.3 & Begin School & 1.2 & Having Children & 2.6 & College & 2.2 \\
\hline 4 . & Job & 2.2 & College & 1.3 & First job & 1.5 & Begin School & 1.2 \\
\hline 5 . & Kindergarten/Daycare & 0.6 & Fall in love & 2.4 & Fall in love & 1.9 & High School & 2.1 \\
\hline 6 . & Puberty & 1.5 & Others' death & -2.6 & College & 2.3 & First job & 1.2 \\
\hline 7 . & Having Children & 2.6 & Retirement & -0.1 & College entr. exam & -0.1 & Begin talking & 2.4 \\
\hline 8 . & Graduation from university & 2.9 & Leave home & 1.1 & Own death & -1.5 & Begin walking & 2.2 \\
\hline 9. & Own Birth & 1.9 & Parents' death & -2.5 & Others' death & -2.8 & Own death & - \\
\hline 10 . & Begin Driving & 2.0 & First job & 1.0 & Puberty & 0.1 & Go to School & 1.8 \\
\hline 11. & Graduation Secondary School & 2.3 & Begin Day Care & 0.3 & Circumcision & 0.5 & Parents' death & 1.8 \\
\hline 12 . & Circumcision & 2.5 & Own death & -0.9 & Begin Talking & 2.5 & Others' death & - \\
\hline 13. & Choosing friends & 0.7 & Divorce & -2.0 & Begin Walking & 2.3 & Retirement & 2.9 \\
\hline 14. & Gender divided social skills & 2.6 & Siblings & 0.6 & Military Service & 0.9 & Own birth & - \\
\hline 15 . & Learn Islamic value \& moral & 2.6 & First friend & 2.9 & Retirement & 0.7 & Fall in love & 2.6 \\
\hline 16. & Raising children & 1.2 & Go to school & 1.2 & Begin high school & 1.8 & Begin driving & 1.6 \\
\hline 17. & Islamic birth rituals & 2.3 & Puberty & -0.2 & Divorce & -2.0 & Grandchildren & 1.4 \\
\hline 18. & Begin walking & 2.8 & Grandchildren & 2.7 & Parents' death & -2.5 & Settle on career & 2.4 \\
\hline 19. & Secondary school/go to school & 1.8 & Long trip & 1.3 & Accident/Injury & -2.1 & Puberty & 2.1 \\
\hline 20 . & Learning to pray & 2.3 & Begin walking & 2.1 & Getting into fights & -1.5 & First sex & 2.5 \\
\hline 21. & Major achievement & 2.8 & Serious Disease & -1.3 & Family quarrels & -1.5 & Leave home & 1.3 \\
\hline 22 . & Own death & -2.3 & Major Achievement & 2.0 & Begin daycare & 1.3 & Begin Daycare & 0.0 \\
\hline 23. & Growing old/Retirement & 0.0 & Settle on Career & 0.7 & High school exam & 0.7 & Empty nest & 1.6 \\
\hline 24. & Women's independence & 1.3 & First sexual experience & 1.5 & Traffic accident & -2.8 & First kiss & 1.6 \\
\hline 25 . & Go to the mosque & 3.0 & Partner's death & -3.0 & Illness & -2.6 & & 2.0 \\
\hline 26 . & Begin talking & 2.8 & Begin talking & 2.2 & Sibling birth & 1.3 & & - \\
\hline 27 . & Death of relative/Others & -2.3 & Confirmation & 2.3 & Move & -1.3 & & 0.8 \\
\hline 28. & Parents help with settling & 2.3 & Enter adulthood & 1.2 & & & & 2.5 \\
\hline 29. & Travel \& studying abroad & 2.0 & Having peers & 2.6 & & & & \\
\hline 30 . & Stability in life & 1.0 & Empty nest & -0.8 & & & & \\
\hline 31. & Moving & 2.5 & First rejection & -3.0 & & & & \\
\hline 32 . & Learn fasting & 1.0 & The "right" job & 3.0 & & & & \\
\hline 33. & HAJJ - pilgrim fair & 3.0 & First contact & 1.5 & & & & \\
\hline 34. & Learning the Quran & 2.5 & Baptism & 1.5 & & & & \\
\hline 35 . & Ennah (trip for young men) & 2.0 & Earn first money & 0.8 & & & & \\
\hline
\end{tabular}


This is an Author's Accepted Manuscript of an article published in Memory (2014), 22:4, 390-407, DOI: 10.1080/09658211.2013.795598 (copyright Taylor \& Francis), available online at: http://www.tandfonline.com/10.1080/09658211.2013.795598 
This is an Author's Accepted Manuscript of an article published in Memory (2014), 22:4, 390-407, DOI: 10.1080/09658211.2013.795598

(copyright Taylor \& Francis), available online at: http://www.tandfonline.com/10.1080/09658211.2013.795598

\section{Table 6}

One way ANOVA showing the characteristics of CLS events displayed by country.

\begin{tabular}{|c|c|c|c|c|c|c|c|c|c|}
\hline \multicolumn{2}{|c|}{ Qatar } & \multicolumn{2}{|c|}{ Denmark } & \multicolumn{2}{|c|}{ Turkey } & \multicolumn{2}{|c|}{ USA } & \multirow[b]{2}{*}{$\mathbf{F}$} & \multirow[b]{2}{*}{$\eta^{2}$} \\
\hline $\mathbf{M}$ & SD & $\mathbf{M}$ & SD & $\mathbf{M}$ & $S D$ & $\mathbf{M}$ & SD & & \\
\hline $1.79^{a}$ & 0.89 & $1.10^{\mathrm{b}}$ & 0.78 & $0.96^{b}$ & 0.92 & $1.53^{\mathrm{a}}$ & 0.72 & $16.08^{* \star *}$ & 0.12 \\
\hline $0.87^{a}$ & 0.92 & $0.39^{b}$ & 0.69 & $0.28^{b}$ & 0.58 & $0.78^{a}$ & 0.99 & $10.66^{* * *}$ & 0.08 \\
\hline $3.42^{a}$ & 1.74 & $4.32^{b}$ & 1.13 & $3.77^{a}$ & 1.62 & $4.59^{b}$ & 1.16 & $11.15^{* * *}$ & 0.09 \\
\hline $1.15^{a}$ & 1.13 & $0.35^{b}$ & 0.54 & $0.71^{\mathrm{a}}$ & 0.84 & $0.04^{c}$ & 0.20 & $34.84^{* \star *}$ & 0.23 \\
\hline $0.65^{a}$ & 0.93 & $0.10^{b}$ & 0.36 & $0.15^{\mathrm{b}}$ & 0.36 & $0.00^{c}$ & 0.00 & $26.64^{\star \star \star}$ & 0.19 \\
\hline $5.35^{a}$ & 4.87 & $24.56^{b}$ & 7.53 & $20.04^{c}$ & 7.41 & $23.07^{b}$ & 6.08 & $23.05^{\star \star \star}$ & 0.17 \\
\hline
\end{tabular}

Note: df range $(3,339-348), * \star \star p<0.001$

Means with different superscripts are significantly different at $\mathrm{p}<0.05$ 
This is an Author's Accepted Manuscript of an article published in Memory (2014), 22:4, 390-407, DOI: 10.1080/09658211.2013.795598 (copyright Taylor \& Francis), available online at: http://www.tandfonline.com/10.1080/09658211.2013.795598

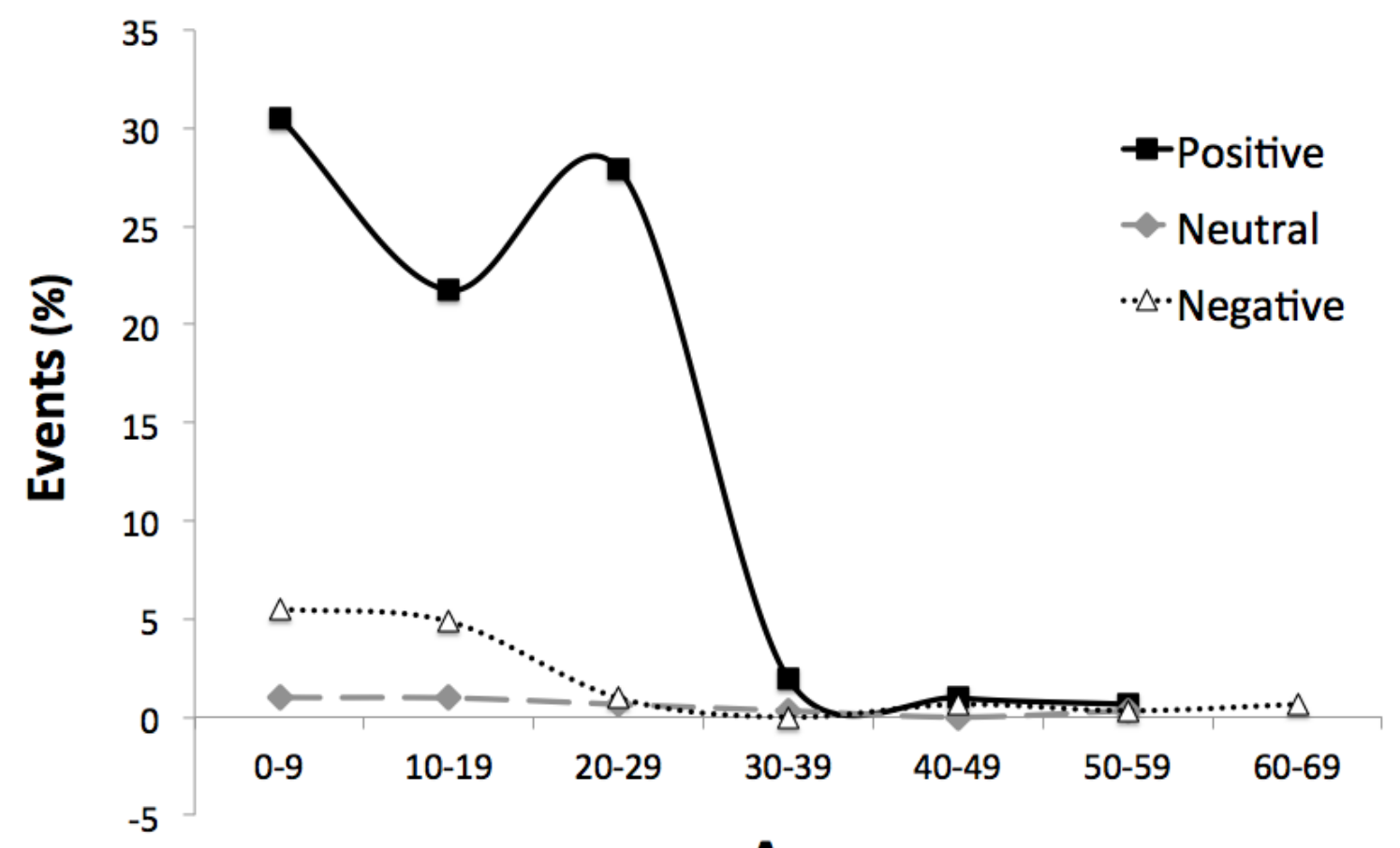

Age

\section{Figure 1}

The Qataris' distribution of age for positive, neutral, and negative CLS events 
This is an Author's Accepted Manuscript of an article published in Memory (2014), 22:4, 390-407, DOI: 10.1080/09658211.2013.795598 (copyright Taylor \& Francis), available online at: http://www.tandfonline.com/10.1080/09658211.2013.795598

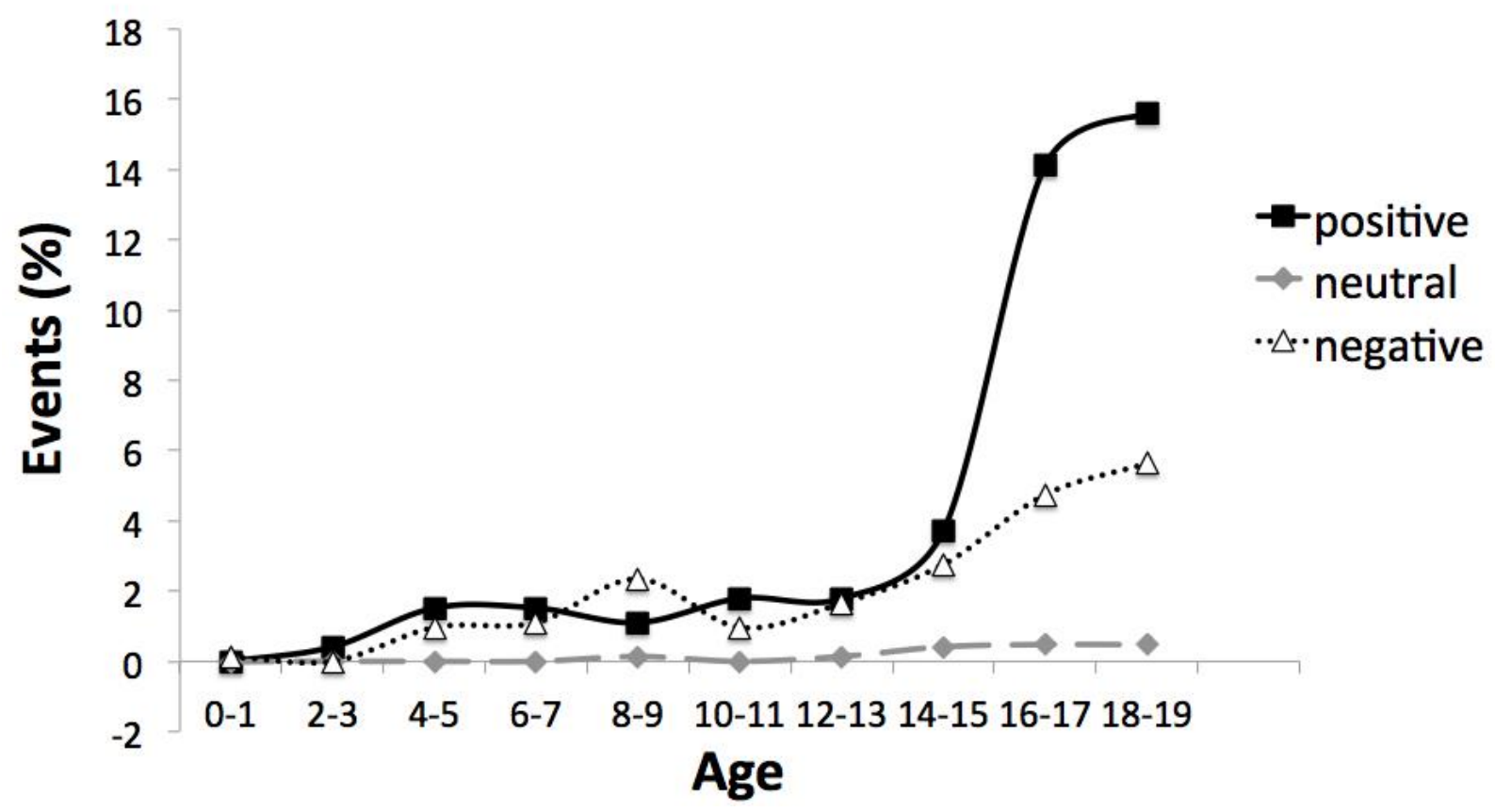

\section{Figure 2}

The Qataris' distribution of age for positive, neutral, and negative life story events in a 2-year interval 
This is an Author's Accepted Manuscript of an article published in Memory (2014), 22:4, 390-407, DOI: 10.1080/09658211.2013.795598 (copyright Taylor \& Francis), available online at: http://www.tandfonline.com/10.1080/09658211.2013.795598

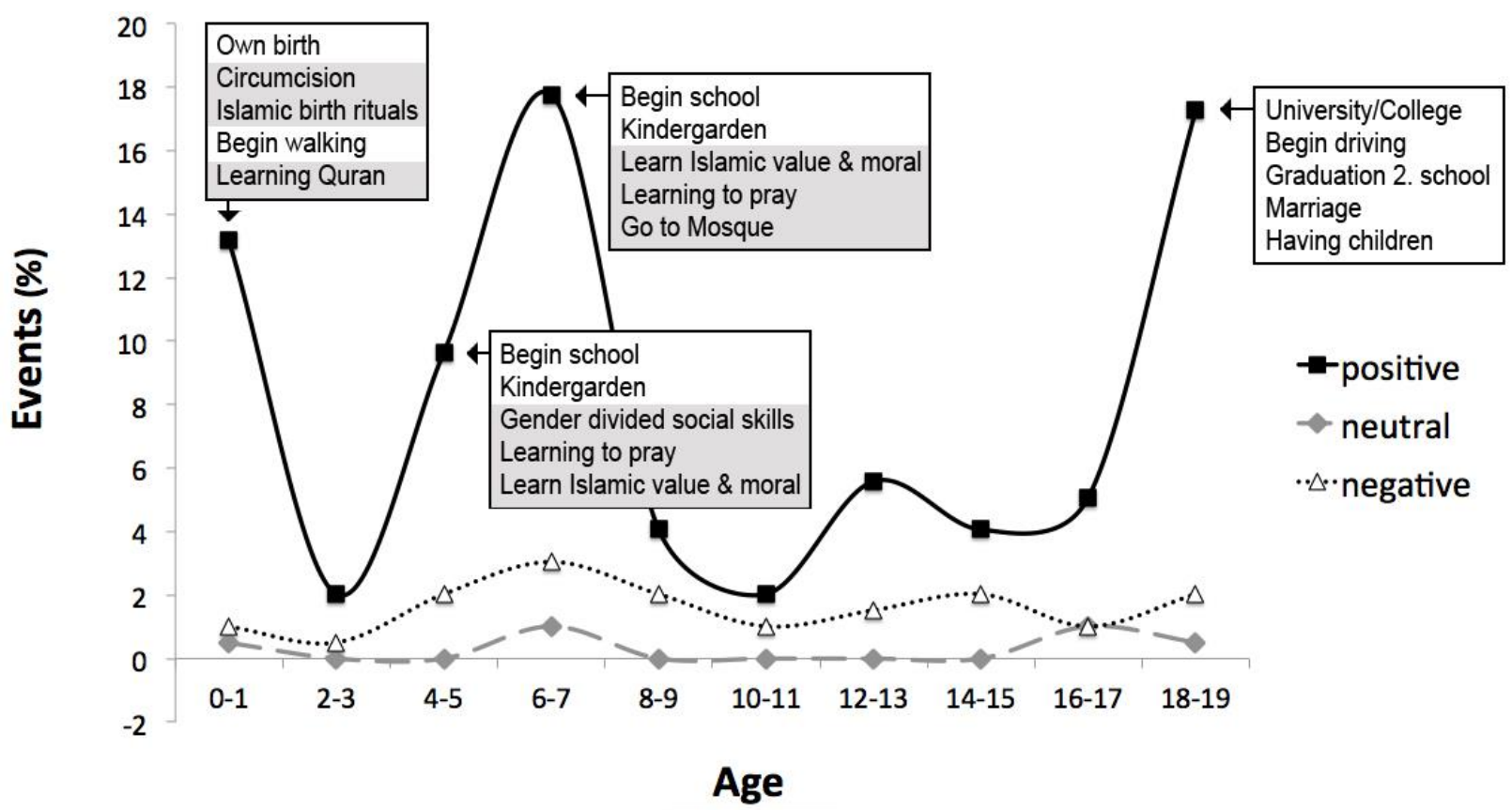

Figure 3

Close up of the first two decades: The Qataris' distribution of age for positive, neutral and negative CLS events in a 2-year interval. The five most prevalent events are shown for the positive bumps and religious events are marked in grey. 
This is an Author's Accepted Manuscript of an article published in Memory (2014), 22:4, 390-407, DOI: 10.1080/09658211.2013.795598 (copyright Taylor \& Francis), available online at: http://www.tandfonline.com/10.1080/09658211.2013.795598

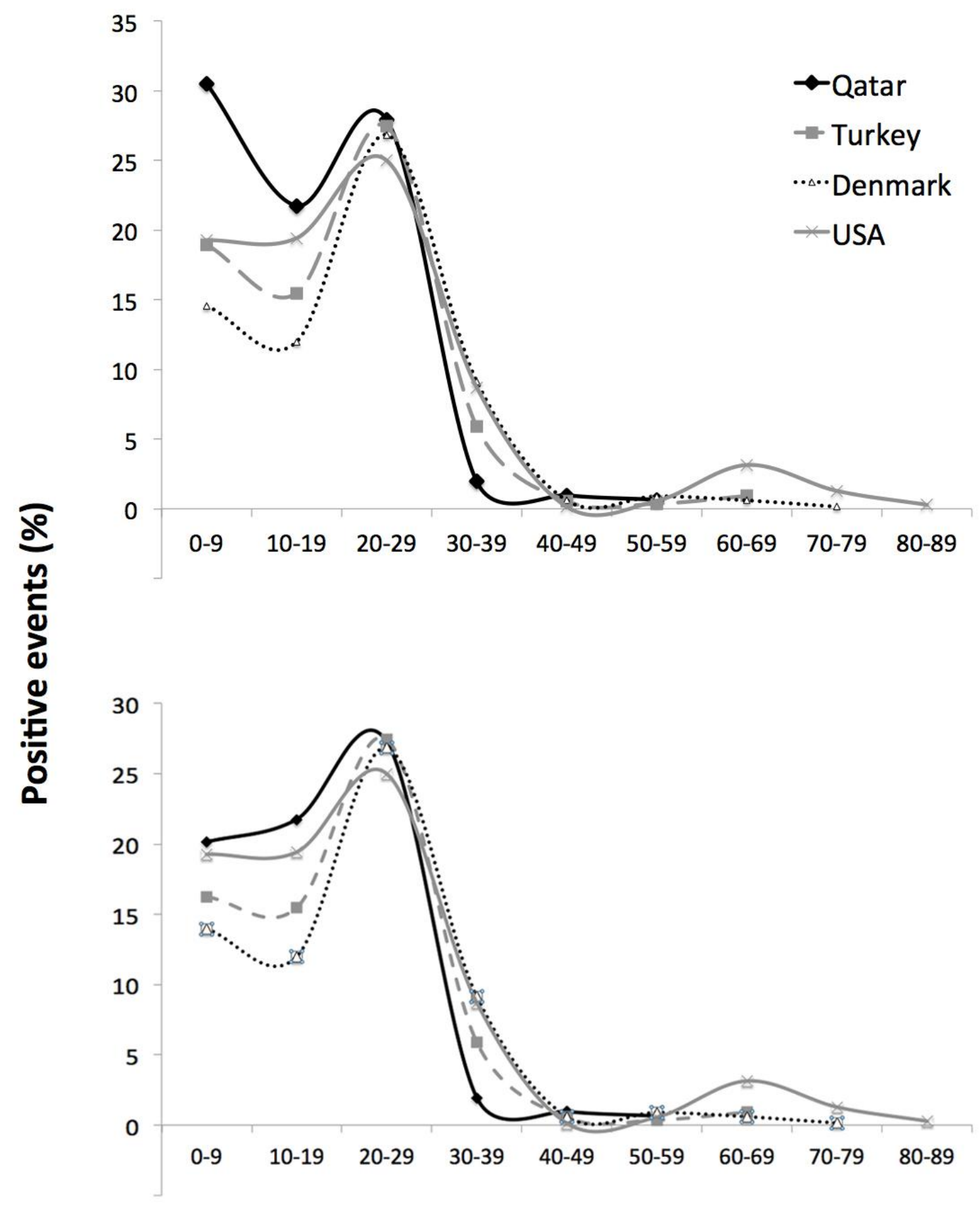

Age

\section{Figure 4}

The top panel shows the distribution of age estimates for all positive CLS events. The bottom panel shows the same distribution excluding all religious events. 